\title{
A GA-based fuzzy resource leveling optimization for helicopter maintenance activity
}

\author{
Malek Masmoudi ${ }^{1}$ Alain Haït ${ }^{1}$ \\ ${ }^{1}$ Université de Toulouse, Institut Supérieur de l'Aéronautique et de l'Espace, Toulouse, France
}

\begin{abstract}
Genetic algorithm is one of the main heuristics that have been applied to scheduling problems in the last few decades. This paper presents a generalization of the genetic algorithm for solving project scheduling problem under time uncertainties within resource leveling technique. The generalization consists of handling fuzzy time parameter and fuzzy resource distribution instead of crisp ones. The provided fuzzy genetic algorithm is justified and applied to a real multi-project and multi-resources problem from the helicopter maintenance activity.
\end{abstract}

Keywords: Helicopter, Maintenance, Fuzzy planning, Genetic Algorithm, Resource Leveling,

\section{Introduction}

Maintenance is a costly and important activity in the aeronautical industry. Aircraft maintenance consists of carrying out all the necessary actions to guarantee the required level of reliability, safety and operational capacity of the aircraft. Hence, the organization of helicopters maintenance, repair and overhaul (MRO) is an important issue and some problems like particularly planning and scheduling have already been studied for the military domain $[20,21]$. In this paper, only Heavy Maintenance Visits (HMV); the heaviest aircraft maintenance checks, are considered. These checks affect all the aspects (structure, avionics, mechanics) and can last up to several months.

The HMV check contains planned maintenance tasks and also corrective maintenance tasks because problems are discovered during the inspection of the helicopter. Precedence constraints exist, due to technical or accessibility considerations. Hence a HMV may be seen as a project involving various resources as operators, equipment and spare parts. Consequently, the management of a maintenance center is viewed as multi-project management, where every project duration should be minimized while respecting capacity constraints.

In[6], multi-project organizations are classified according to projects variability and dependency. Dealing with high variability is an important issue in aircraft maintenance domain. Although it is qualified as non optimal, the Theory of Constraints provided by Eliyahu Goldratt has proven its effectiveness when coping with scheduling problem under uncertainty in military aircraft maintenance [19]. According to our knowledge, no solution has been provided in literature for civil helicopter maintenance. This can be explained by the dispersal of civil actors who are positioning as small service providers with limited resources. Helicopters have some specificities: particular flight conditions, compact volume, high criticality etc. Moreover, in contrary to military helicopter maintenance workshops, a great heterogeneity is involved when repairing civil helicopters.

Focused on civil domain, three high sources of uncertainty have been identified: uncertainty on the release date, the inspection duration and the procurement delays. To cope with these uncertainties on scheduling optimization, a fuzzy set modeling has been justified and explained in[14].

Typically, two techniques - time driven and resource driven - are employed to solve project scheduling problem. In this paper we focus on the first technique and we provide a new generalization of genetic algorithm to fuzzy Resource Leveling Problem.

The paper is organized as follows. The second section introduces Fuzzy set theory and its application to projects modeling. In the third section, a GA procedure to solve resource leveling problem is described. In the forth section, a generalization of the GA-based resource leveling technique to fuzzy time and resource parameters is provided. The last section contains some conclusions.

\section{Fuzzy time and resource modeling}

\subsection{Fuzzy set theory}

Introduced by Zadeh [16] in 1965, The fuzzy set theory is well-suited to cope with uncertainties. It becomes more and more useful in different domains especially in production management $[3,12]$.

Zadeh has defined a fuzzy set $\widetilde{A}$ as a subset of a referential set $X$, whose boundaries are gradual rather than abrupt. Thus, the membership function $\mu_{\widetilde{A}}$ of a fuzzy set assigns to each element $x \in X$ its degree of membership $\mu_{\widetilde{A}}(x)$ taking values in $[0,1]$.

Many profiles are used in the literature to model fuzzy quantity. Particularly, the trapezoidal profile (Fig.1) is the best-supported by the possibility approach (Sec. 2.3). 


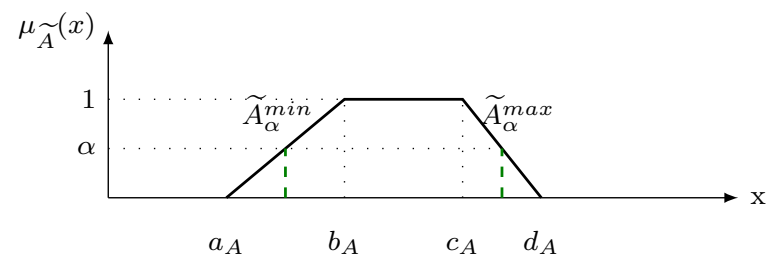

Figure 1: Trapezoidal fuzzy set

To generalize some operations from classical logic to fuzzy sets, Zadeh has given the possibility to represent a fuzzy profile by an infinite family of intervals called $\alpha$-cuts. Hence, the fuzzy profile $\widetilde{A}$ can be defined as a set of intervals $A_{\alpha}=\left\{x \in X ; \mu_{\widetilde{A}}(x) \geq\right.$ $\alpha\}$ with $\alpha \in[0,1]$. It became consequently easy to utilize classical interval arithmetics and adapt it to fuzzy numbers. Dubois and Prade [3] and Chen and Hwang [10] have defined mathematical operations that can be performed on trapezoidal fuzzy sets. Let $\widetilde{A}\left(a_{A}, b_{A}, c_{A}, d_{A}\right)$ and $\widetilde{B}\left(a_{B}, b_{B}, c_{B}, d_{B}\right)$ be two trapezoidal fuzzy numbers, then:

$$
\widetilde{A} \oplus \widetilde{B}=\left(a_{A}+a_{B}, b_{A}+b_{B}, c_{A}+c_{B}, d_{A}+d_{B}\right),
$$

$$
\widetilde{A} \ominus \widetilde{B}=\left(a_{A}-d_{B}, b_{A}-c_{B}, c_{A}-b_{B}, d_{A}-a_{B}\right),
$$

$$
\begin{array}{r}
\max (\widetilde{A}, \widetilde{B})=\left(\max \left(a_{A}, a_{B}\right), \max \left(b_{A}, b_{B}\right),\right. \\
\left.\max \left(c_{A}, c_{B}\right), \max \left(d_{A}, d_{B}\right)\right)
\end{array}
$$

$$
\begin{aligned}
\min (\widetilde{A}, \widetilde{B})=( & \min \left(a_{A}, a_{B}\right), \min \left(b_{A}, b_{B}\right), \\
& \left.\min \left(c_{A}, c_{B}\right), \min \left(d_{A}, d_{B}\right)\right)
\end{aligned}
$$

Other operations like multiplication and division have also been studied. For more details on fuzzy arithmetics we refer readers to [3].

\subsection{Fuzzy PERT technique}

The PERT/CPM technique is used to determine critical tasks in a project. This technique is composed of two successive steps: The forward propagation providing earliest starting and finishing dates and consequently free margins and the Backward propagation providing latest starting and finishing dates and consequently total margins.

PERT technique was studied in case of fuzzy time parameters and recently some complexities were raised $[1,15]$. In fact, contrary to forward propagation that can faultless be easily generalized to fuzzy parameters by applying formulas 1 and 4, the generalization of backward propagation by using formulas 2 and 3 would provide erroneous or even negative values because uncertainty would be taken into account twice [1]. Soltani and Haji [15] suggest a modification of the back propagation by eliminating negative values using a PL-based method. By applying this method, the domain of possible values is restricted. Dubois et al. [1] show that the boundaries of latest dates (and consequently floats) are reached in extreme configurations. They suggest some algorithms based on properties defined by Dubois et al. [2] to calculate optimally the fuzzy latest starting times and fuzzy total floats. These algorithms are applied in this work and some formulas are added (Sec. 4.1) to calculate the fuzzy latest finishing times and other fuzzy time parameters useful for the resource leveling problem.

\subsection{Possibility theory}

To cope with decision making on fuzzy area, Zadeh [17] developed the concept of the possibility approach based on fuzzy subsets. Recently, possibility theory becomes frequently used in fuzzy scheduling problems $[9,22]$.

The possibility theory introduces both a possibility measure (denoted $\Pi$ ) and a necessity measure (denoted $N$ ). Let $P$ be a set (fuzzy or not) and $\widetilde{A}$ a fuzzy set attached to a single valued variable $t$. The possibility of the event " $t \in P$ ", denoted by $\Pi(t \in P)$, evaluates the extent to which the event is "possibly" true. It is defined as the degree of intersection between $\widetilde{A}$ and $P$ by the minimum operation:

$$
\Pi(t \in P)=\sup _{u} \min \left(\mu_{\widetilde{A}}(u), \mu_{P}(u)\right)
$$

The dual measure of necessity of the event " $t \in$ $P$ ", denoted by $N(t \in P)$, evaluates the extent to which the event is "necessarily true". It is defined as the degree of the inclusion $(\widetilde{A} \subset P)$ by the maximum operation:

$$
\begin{aligned}
N(t \in P) & =\inf _{u} \max \left(1-\mu_{\widetilde{A}}(u), \mu_{P}(u)\right) \\
& =1-\Pi\left(t \in P^{c}\right)
\end{aligned}
$$

Where $P^{c}$ is the complementary of $P\left(\mu_{P^{c}}(u)=\right.$ $\left.1-\mu_{P}(u)\right)$.

Let $t$ be a real-valued variable in the fuzzy interval $\widetilde{A}$ and $\tau$ be a constant. To measure the truth of the event $\widetilde{A}>\tau$, equivalent to $t \in[\tau ;+\infty)$, we need the couple $\Pi(\tau \leq t)$ and $N(\tau \leq t)$ (Fig. 2). Thus :

$$
\begin{aligned}
\Pi(\tau \leq t) & =\mu_{(-\infty ; \widetilde{A}]}(\tau)=\sup _{u \geq \tau} \mu_{\widetilde{A}}(u) \\
& =\sup _{u} \min \left(\mu_{\widetilde{A}}(u), \mu_{[\tau ;+\infty)}(u)\right)
\end{aligned}
$$


and

$$
\begin{aligned}
N(\tau \leq t) & =\mu_{(-\infty ; \widetilde{A}[}(\tau)=\inf _{u<\tau}\left(1-\mu_{\widetilde{A}}(u)\right) \\
& =\inf _{u} \max \left(1-\mu_{\widetilde{A}}(u), \mu_{[\tau ;+\infty)}(u)\right)
\end{aligned}
$$

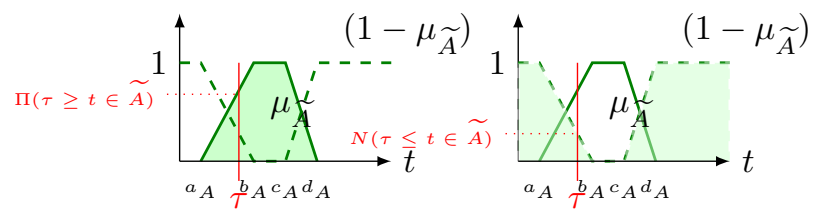

Figure 2: Necessity and possibility of $\tau \leq t \in \widetilde{A}$.

Consequently, let $\tau$ and $\sigma$ two variables in respectively fuzzy intervals $\widetilde{A}$ and $\widetilde{B}$ and $t$ a real value. To measure the truth of the event " $t$ between $\widetilde{A}$ and $\widetilde{B}$ " we need both $\Pi(\widetilde{A} \leq t \leq \widetilde{B})$ and $N(\widetilde{A} \leq t \leq \widetilde{B})$. Thus:

$$
\begin{aligned}
\Pi(\widetilde{A} \leq t \leq \widetilde{B}) & =\mu_{[\widetilde{A} ; \widetilde{B}]}(t)=\mu_{[\widetilde{A} ;+\infty) \cap(-\infty ; \widetilde{B}]}(t) \\
& =\min \left(\mu_{[\widetilde{A} ;+\infty)}(t), \mu_{(-\infty ; \widetilde{B}]}(t)\right)
\end{aligned}
$$

and

$$
\begin{aligned}
N(\widetilde{A} \leq t \leq \widetilde{B}) & =\mu_{] \widetilde{A} ; \widetilde{B}[}(t)=\mu_{] \widetilde{A} ;+\infty) \cap(-\infty ; \widetilde{B}[}(t) \\
& =\min \left(\mu_{] \widetilde{A} ;+\infty)}(t), \mu_{(-\infty ; \widetilde{B}[}(t)\right)
\end{aligned}
$$

\subsection{Fuzzy workload}

The workload plan is established and compared to the available capacity to decide the feasibility of a project schedule. In the literature, the majority of authors who work with fuzzy sets in scheduling problems apply $\alpha$-cuts on fuzzy dates. Hence, they generate a set of deterministic optimistic and pessimistic workload plans $[7,8,13]$.

In [14], the authors use possibility theory to define the new concept of fuzzy workload distribution. They generate a unique couple of fuzzy optimistic and pessimistic workload plans.

Let $T$ be an operational task and $\widetilde{S}\left(a_{S}, b_{S}, c_{S}, d_{S}\right)$ its fuzzy starting date, $\widetilde{F}\left(a_{F}, b_{F}, c_{F}, d_{F}\right)$ its fuzzy finishing date, $\widetilde{D}(w, x, y, z)$ its fuzzy duration and $r$ its required resources. For each period, four values are given, corresponding to scenarios with the four duration values $w, x, y, z$ of the tasks. Hence, the workload plan can be represented as suggested by Grabotet al. [22] for fuzzy MRP. Masmoudi and Haï [14] define the new concepts of necessary workload $N$ and possible workload $\Pi$ and between particularly the pessimistic workload distribution $p^{*}$ and

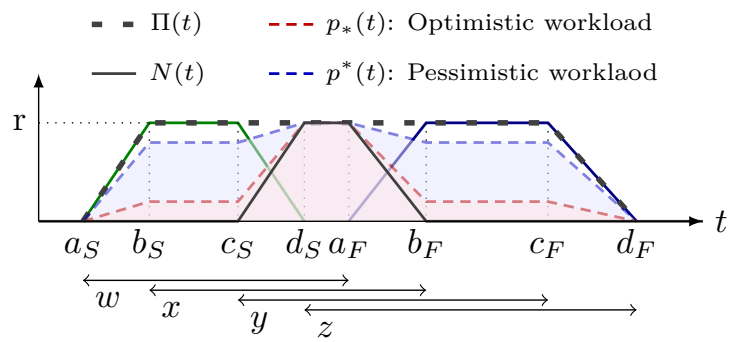

Figure 3: Fuzzy resource profiles.

the optimistic workload distribution $p_{*}$ corresponding respectively to the extreme tasks durations $\mathrm{w}$ and z (Fig. 3).

The Fig. 4 shows an example of optimistic and pessimistic workload plans built for a small project with three tasks $T_{i}(i \in\{1,2,3\})$. The correspondent resource requirement are $r_{1}=r_{2}=2$ and $r_{3}=1$. For each task $T_{i}$, pessimistic and optimistic workload are established (dashed lines). The Integration of all tasks profiles over periods of time gives the mean resource workload $R_{i}$ of the project for pessimistic and optimistic cases (Fig. 4).

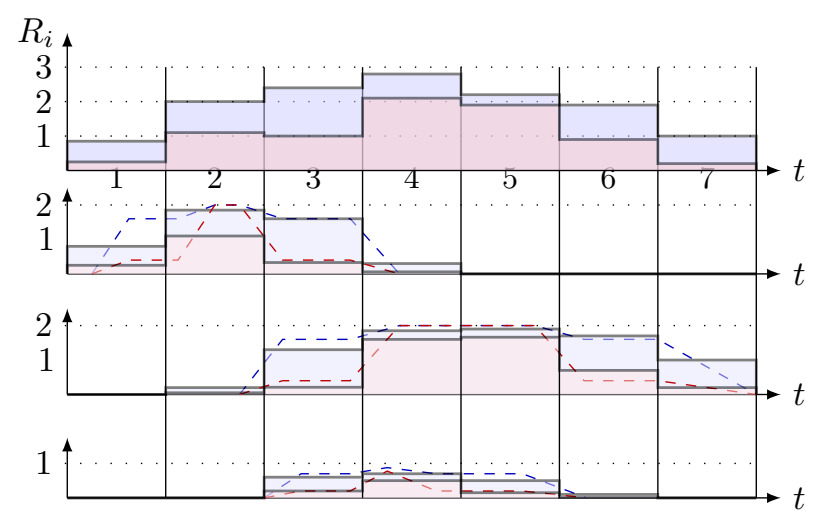

Figure 4: Optimistic and pessimistic workload Plans

The concept of pessimistic and optimistic fuzzy distributions is adopted in the generalization of the resource leveling technique using GA to fuzzy parameters.

\section{Genetic Algorithm for resource leveling}

Many analytical and heuristic methods were developed to solve resource leveling problems [4, 13, 18]. The GA is particularly studied in this paper. The following section presents first some literature about the Genetic Algorithm. Then an adopted GA to the problem of resource leveling is provided. This GA will finally generalized to fuzzy parameters.

\subsection{Literature about Genetic Algorithm}

A genetic algorithm (GA) is a search heuristic that follows the natural evolutionary process. The 
strength of the Genetic algorithm is that it represents the main computational intelligence approach that cope with big instances. Hence, since 1975, the GA has been used to solve complex problems like particularly the multi-projects and multi-objectives scheduling problem [11]. The technique of GA is quite known thus it will not be explained in detail in this paper. Hence, to get more complete information about the Genetic Algorithm technique we refer readers to [5].

\subsection{Genetic Algorithm description}

In multi-projects context, the Resource Leveling Problem can be defined as a set of tasks with precedence constraints and predetermined durations. A schedule is defined by a set of tasks' starting times. Let $n$ the total number of tasks and let $P$ be the number of projects to schedule and $n_{j}$ the number of tasks in project $j$ $\left(n=\sum_{j=1}^{P} n_{j}\right)$. A schedule is defined by the set $S=\left(S_{11}, S_{21}, \ldots, S_{n_{1} 1}, \ldots, S_{i j}, \ldots, S_{1 P}, \ldots, S_{n_{P} P}\right)$ where $S_{i j}$ is the starting time of the task $i$ from the project $j(\operatorname{Card}(S)=n)$.

The CPM technique is applied to a scheduling problem without resources consideration in order to define the lower and upper bounds of each value $S_{i j}$ which are respectively the Earliest Starting time $\left(E S_{i j}\right)$ and the Latest Starting time $\left(L S_{i j}\right)$ of the task $i$ from the project $j$.

The objective $L$ is to smooth resources utilization which can be mathematically expressed as follows:

$$
\min \left(\sum_{k=1}^{K}\left(\sum_{t=1}^{T} \sum_{j=1}^{P} \sum_{i=1}^{n_{j}}\left(r_{k i t}-\left[\sum_{t=1}^{T} \sum_{j=1}^{P} \sum_{i=1}^{n_{j}} r_{k i t}\right] / D\right)^{2}\right)\right)
$$

Where:

$L$ : The resource leveling index indicates the sum of squared differences between period resource usage and average resource usage.

$r_{k i j t}$ : The partial resource $k$ demand of the activity $i$ from the project $j$ at the period of time $t$.

$D$ : The projects duration.

$K$ : The number of resource types.

$P$ : The number projects.

$n_{j}$ : The number tasks in project $j$.

The genetic algorithm procedure followed in this paper to cope with resource leveling problem is presented below in pseudo-code form:

A GENETIC ALGORITHM procedure for resource leveling problem:

Apply CPM/PERT technique to have for each task its Earliest Starting and Latest Starting times

Parametrize the Genetic algorithm;

\section{Begin $G A$ :}

Generate initial population $P_{0}$ of $n_{\text {pop }}$ candidates;

Evaluate initial population $P_{0}$;

Initialize generation counter $t \leftarrow 0$;

While Stopping criteria not satisfied Repeat:
Select $m$ best candidates; The best $m_{n}$ candidates from $m$ are identically kept for $P_{t+1}$ and the $m \backslash m_{n}$ other candidates are reproduced based on Elitist method until the population $P_{t+1}$ is completely generated;

Crossover $m_{k}$ candidates (from $n_{p o p} \backslash m_{n}$ ) randomly at one or more random position(s);

Mutate $m_{d}$ candidates (from $n_{\text {pop }} \backslash m_{n}$ ) randomly, by mutating gmut random genes per candidate;

Evaluate new population $P_{t+1}$ and select the best candidate for the iteration;

Increment current population: $P_{t} \leftarrow P_{t+1}$;

Increment generation counter: $t \leftarrow t+1$;

\section{End while;}

\section{End GA;}

The issue of applying Genetic Algorithm is to select an appropriate form of the chromosome representation. In resource leveling problem, the wellappropriate form is the one considering starting times of tasks as decision variable being coded as genes values. Thus, the sequence of the tasks in the chromosome corresponds to the sequence of tasks project by project sorted by their Id number. Each gene value is equal to a possible starting time of corresponding task. The starting time of each task $T_{i j}$ is chosen randomly in its domain rate respecting precedence constraints.

\begin{tabular}{|c|c|c|c|c|c|c|c|c|c|c|}
\hline \multirow[t]{2}{*}{ Task's Id } & \multicolumn{4}{|c|}{ Project 1} & \multicolumn{6}{|c|}{ Project $P$} \\
\hline & $T_{11}$ & $T_{21}$ & $\ldots$ & $T_{n 1}$ & & $T_{i j}$ & $\ldots$ & $T_{1 P}$ & $\ldots$ & $T_{n P}$ \\
\hline & $S_{11}$ & $S_{21}$ & $\ldots$ & $s_{n 1}$ & $\cdots$ & $S_{i j}$ & $\cdots$ & $S_{1 P}$ & $\cdots$ & $S_{n P}$ \\
\hline
\end{tabular}

Task's starting time

Figure 5: Chromosome representation in Multiproject resource leveling

The fitness function needed to evaluate chromosomes is chosen equal to the resource leveling index $L$ defined in 11 . There was no need in our algorithm to modify the equation to cope with the fact that GA is traditionally designed to solve problems of seeking maximums.

The adopted selection technique is the roulette wheel method that we combine with Elitist method [5] in order to improve selection efficiency. Thus, the selection probability for a chromosome $k$ is proportional to the ratio $f_{k} / \sum_{j=1}^{n_{\text {pop }}} f_{j}$, where $f_{k}$ is the fitness value of the chromosome $k$ and $n_{\text {pop }}$ is the population size. According to the Elitist method, the bests chromosomes of the current generation are kept and preserved into the next generation.

The GA operators are uniform 1-point crossover and uniform mutation. The crossover starts with randomly selecting a cut point and parent's chromosomes. The right parts of the chromosomes are swapped and hence child are generated(Fig. 6). Some child generated with this way do not satisfy 


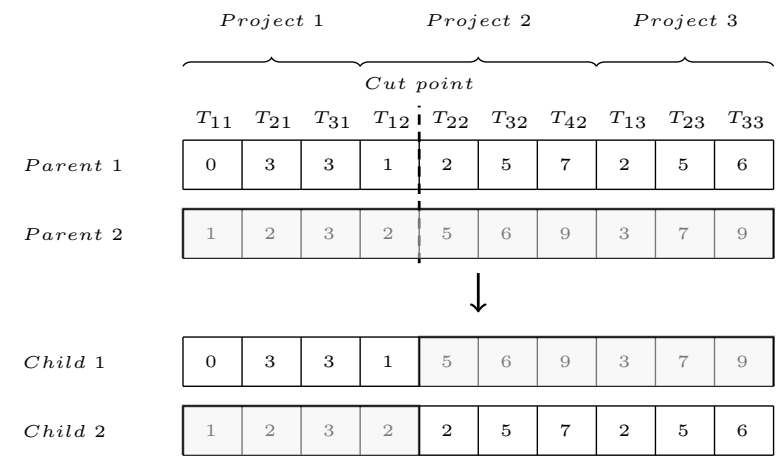

Figure 6: Uniform 1-point crossover

precedence constraints. To deal with this situation, a reparation technique is applied (Fig. 7).

\begin{tabular}{|c|c|c|c|c|c|c|c|c|c|c|}
\hline & \multicolumn{3}{|c|}{ Project 1} & \multicolumn{4}{|c|}{ Project 2} & \multicolumn{3}{|c|}{ Project 3} \\
\hline & $T_{11}$ & $T_{21}$ & $T_{31}$ & $T_{12}$ & $T_{22}$ & $T_{32}$ & $T_{42}$ & $T_{13}$ & $T_{23}$ & $T_{33}$ \\
\hline Parent & 1 & 2 & 3 & 2 & 2 & 5 & 7 & 2 & 5 & 6 \\
\hline bild & 1 & 2 & 3 & 2 & 3 & 5 & 8 & 2 & 5 & 6 \\
\hline
\end{tabular}

Figure 7: Reparation after crossover

Let $k$ the one-cut-point value and the task $T_{i j}$ is the correspondent task of the gene $k$. All genes values of the successors of $k$ must be checked to deal with precedence constraints. Hence, the task $k+1$ is the first task to be checked if its part of the project $j$, else no reparation is needed. The formula of reparation is the following:

$$
\begin{gathered}
S_{l j}=\max \left(S_{l j}, \max _{p \in \operatorname{pred}\left(T_{l j}\right)}\left(S_{p j}+D_{p j}\right)\right) \\
\forall l \in[i+1, n]
\end{gathered}
$$

Where:

$\operatorname{pred}\left(T_{l j}\right)$ : The set of predecessors of task $T_{l j}$.

$D_{p j}$ : The duration of the task $T_{p j}$.

The mutation consists of randomly replacing at least one gene with a random real value within the specified range of the corresponding task's starting

\begin{tabular}{|c|c|c|c|c|c|c|c|c|c|c|}
\hline & $T_{11}$ & $T_{21}$ & $T_{31}$ & $T_{12}$ & $T_{22}$ & $T_{32}$ & $T_{42}$ & $T_{13}$ & $T_{23}$ & $T_{33}$ \\
\hline Parent & 1 & 2 & 3 & 2 & 3 & 5 & 8 & 2 & 5 & 6 \\
\hline & 1 & 2 & 3 & 1 & 3 & 5 & 8 & 2 & 5 & 6 \\
\hline
\end{tabular}
time (Fig. 9).

Figure 8: Uniform mutation

Let $k$ a selected gene to mutate and the task $T_{i j}$ is its correspondent task. The new value of the gene is chosen randomly between the maximum finishing time of predecessors tasks $\left(\max _{p \in \operatorname{pred}\left(T_{i j}\right)}\left(S_{p j}+\right.\right.$ $\left.D_{p j}\right)$ )and the minimum starting time of successors $\operatorname{tasks}\left(\min _{p \in \operatorname{succ}\left(T_{i j}\right)}\left(S_{p j}\right)\right)$.

\section{Fuzzy genetic algorithm for resource leveling}

Resource Leveling technique for Fuzzy Scheduling Problem is studied in some recent papers $[13,18]$ where genetic algorithm is adopted to projects with fuzzy time parameters. The idea in these papers is to make a different $\alpha$-cuts on tasks durations to obtain pessimistic and optimistic scenarios for each $\alpha$ cut and then apply deterministic Genetic Algorithm to each scenario to find the correspondent best plan.

In this section a new vision of fuzzy resource leveling is provided. The idea is to make just one couple of fuzzy Genetic Algorithm instead of multideterministic ones. Therefore, some useful hypothesis and extensions are suggested.

\subsection{Useful fuzzy concepts}

Genetic Algorithm developed in (Sec. 3.2) well cope with deterministic Mutli-projects and Multi resources scheduling problems. To be generalized to fuzzy parameters some useful fuzzy concepts are considered.

A trapezoidal fuzzy number is numerically represented by 4 deterministic values (Sec. 2.1). Genetic algorithm becomes very heavy in compilation when considering 4 numbers for each fuzzy decision variable. To deal with this problem only one value is considered and then the encoding and decoding of each solution (chromosome)is done according to the principal of linearity (Fig. 9) which appears logical in our case.

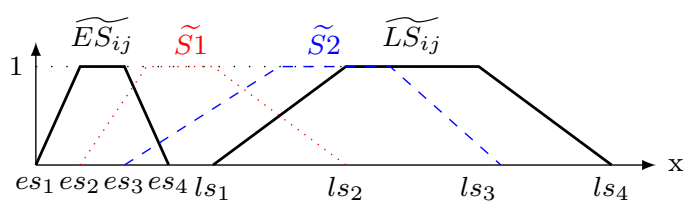

Figure 9: Linearity

Let $\widetilde{E S_{i j}}=\left[e s_{1}, e s_{2}, e s_{3}, e s_{4}\right]$ the Earliest starting time and $\widetilde{L S_{i j}}=\left[l s_{1}, l s_{2}, l s_{3}, l s_{4}\right]$ the Latest starting time of task $T_{i j}$. To generate a possible Starting time $\widetilde{S_{i j}}=\left[s_{1}, s_{2}, s_{3}, s_{4}\right]$, we choose randomly a value of $s_{4}$ between $e s_{4}$ and $l s_{4}$. Let $\beta=\left(s_{4}-l s_{4}\right) /\left(e s_{4}-l s_{4}\right)$. Thus, $\widetilde{S_{i j}}$ is simply calculated according to the principle of linearity within $s_{i}=\beta e s_{i}+(1-\beta) l s_{i} \forall i \in\{1,2,3\}$. In Fig. 9 two examples of possible starting times are showed; $\widetilde{S 1}$ with choosing $\beta=1 / 3$ and $\widetilde{S 2}$ with choosing $\beta=2 / 3$.

Some algorithms in [1] are provided to well calculate fuzzy latest starting times and fuzzy total 
floats. However, no algorithms are provided in the same framework to calculate fuzzy latest finishing times. As these parameters are necessary for our study, the following formula is provided to calculate them:

$$
\widetilde{L F_{i j}}=\min \left(\widetilde{L S_{i j}}+\widetilde{D_{i j}}, \min \left(\widetilde{L S_{s u c c}(i j)}\right), \widetilde{D d(j)}\right)
$$

Where:

$L_{i j}$ : The fuzzy Latest Finishing time of task $T_{i j}$.

$\widetilde{D d_{j}}$ : The fuzzy duedate of the project $j$.

As latest starting times are calculated within the consideration of extreme configuration as explained in [2], the value of $\widetilde{L S_{i j}}+\widetilde{D_{i j}}$ can exceed the range domain of $\widetilde{L F_{i j}}$. In fact, the duration $\widetilde{D_{i j}}$ of the task $T_{i j}$ is not necessarily totally in the range of the extreme configurations provided by the forward propagation. Thus, the 13 provide meaningful computable results respecting precedence constraints. Considering the same explanation, the finishing time is calculated as follows:

$$
\widetilde{F_{i j}}=\min \left(\widetilde{S_{i j}}+\widetilde{D_{i j}}, \widetilde{L F_{i j}}\right)
$$

Once starting and finishing times are calculated for each task, fuzzy workload is established as explained in Sec. 2.4. Thus, for each solution (chromosome), the corresponding fuzzy fitness $\widetilde{L}$ is calculated as follows:

$$
\min \left(\sum_{k=1}^{K}\left(\sum_{t=1}^{T} \sum_{j=1}^{P} \sum_{i=1}^{n_{j}}\left(\widetilde{r}_{k i t}-\left[\sum_{t=1}^{T} \sum_{j=1}^{P} \sum_{i=1}^{n_{j}} \widetilde{r}_{k i t}\right] / \widetilde{D}\right)^{2}\right)\right)
$$

Many defuzzification techniques are provided in literature $[23,24]$ to cope with fuzzy rules particularly while using Genetic Algorithm [25]. These techniques can certainly be applied to defuzzify our fitness function. Nevertheless, in this work, we only consider the extreme values while durations are equal to $w$ or $z$ to get the corresponding optimistic and pessimistic workload plans. Moreover $\widetilde{D}$ is always projected to the maximum value of the projects duration.

\subsection{Real case study from Helicopter maintenance domain}

The Fuzzy Genetic Algorithm is applied to a real RCCP from the helicopter maintenance domain. In MRO, three principal human resources are needed to make HMVs; avionics, structure and mechanics experts. They work generally on more than one helicopter at the same time. Table1 contains the data of a real example with three fuzzy projects.

The GA parameters values are fixed as follows:

- $n_{p o p}$ : Population Size $\left(n_{p o p}=100\right)$.
- $m$ : The best candidates to reproduce $(m=$ $\left.\operatorname{round}\left(n_{\text {pop }} / 3\right)\right)$.

- $m_{n}$ : The best of the best candidates $\left(m_{n}=\right.$ $\left.\max \left(2, n_{\text {pop }} / 20\right)\right)$.

- $m_{k}$ : The number of candidate to crossover $\left(m_{k}=2 * \operatorname{round}\left(2 *\left(n_{\text {pop }}-m_{n}\right) / 5\right)\right)$.

- $m_{d}$ : Number of candidate to mutate $(d=$ $\left.\operatorname{round}\left(3 *\left(n_{\text {pop }}-m_{n}\right) / 5\right)\right)$.

- gmut: Number of genes to mutate by candidate $(\operatorname{gmut}=\min (2, \operatorname{round}(n / 10)))$.

- $n_{\text {iter }}$ : Number of iterations $\left(n_{\text {iter }}=60\right)$.

- $n_{\text {stop }}$ : Stop algorithm condition (with $n_{\text {stop }}=$ 5 , if the result is the same for 5 successive iterations then stop algorithm).

The Genetic Algorithm is applied twice; for pessimistic and for optimistic case separately. Fig. 10 shows the earliest planning, the results and the convergence of the GA for the two cases.

\section{Conclusion}

This paper presents a generalized heuristic for solving scheduling problem under time uncertainties modeled by fuzzy memberships. A new Genetic Algorithm is developed to cope with fuzzy multi-resources and multi-projects Resource Leveling Problem. This algorithm is based on the idea of fuzzy workload plan developed in [14]. Contrary to the classical method existing in the literature which consists of applying $\alpha$-cuts on fuzzy parameters to generate multi deterministic couples of pessimistic and optimistic workload plans, this new generalized algorithm provides one couple of pessimistic and optimistic fuzzy workload plans. It is applied to a real work case and gives interesting results. The Idea of integrating this algorithms into a DSS is under development for a real MRO.

\section{References}

[1] D. Dubois and H. Fargier and J. Fortin, Computational methods for determining the latest starting times and floats of tasks in intervalvalued activity networks, Journal of Intelligent Manufacturing, 16:407-421, 2005.

[2] D. Dubois and H. Fargier and V. Galvagnon, On latest starting times and floats in activity networks with ill-known durations, European Journal of Operational Research, 147:2:266-280, 2003.

[3] D. Dubois and H. Prade, Possibility theory: an approach to computerized processing of uncertainly, International Journal of General Systems, New York, 1988.

[4] S. M. Easa, Resource leveling in construction by optimization, Journal of Construction Engineering and Management, ASCE, 115:2:302316, 1989.

[5] D. E. Goldberg, Genetic Algorithms in Search, Optimization and Machine Learning, 1st, 


\begin{tabular}{|c|c|c|c|c|c|c|}
\hline Tasks Name & Id & Project 1 & Project 2 & Project 3 & Predecessors & Resources \\
\hline & & Duration (periods) & Duration(periods) & Duration(periods) & & $\mathrm{R} 1-\mathrm{R} 2-\mathrm{R} 3$ \\
\hline Waiting for the release date & A & {$[7,8,9,10]$} & {$[10,11,12,13]$} & {$[17,18,19,20]$} & & $0-0-0$ \\
\hline First check when receiving the helicopter & B & [1] & [1] & [1] & A & $2-0-0$ \\
\hline Removal structural and mechanical parts & $\mathrm{C}$ & [3] & [3] & [3] & $\mathrm{B}$ & $3-0-0$ \\
\hline Removal avionics & $\mathrm{D}$ & [3] & [3] & [3] & B & $0-1-0$ \\
\hline Supplying procedure for finishing & $\mathrm{E}$ & {$[13,14,15,16]$} & {$[11,12,13,14]$} & {$[11,12,13,14]$} & $\mathrm{C}$ & $0-0-0$ \\
\hline First part of mechanical inspection & $\mathrm{F}$ & [5] & [5] & {$[3]-$} & $\mathrm{C}$ & $1-1-0$ \\
\hline Supplying procedure for assembling task & G & {$[5,7,8,8]$} & {$[4,5,7,7]$} & {$[5,5,7,7]$} & $\mathrm{C}$ & $0-0-0$ \\
\hline Supplying procedure during structural inspection & $\mathrm{H}$ & {$[2,2,2,3]$} & {$[1,2,2,3]$} & {$[2,3,4,4]$} & $\mathrm{C}$ & $0-0-0$ \\
\hline Subcontracted structure-cleaning & I & {$[1]$} & {$[1]$} & {$[1]$} & $\mathrm{C}$ & $0-0-0$ \\
\hline Subcontracted avionic tests and repairs & $\mathrm{J}$ & {$[2,3,4,5]$} & {$[2,3,4,5]$} & {$[2,3,4,5]$} & $\mathrm{D}$ & $0-0-0$ \\
\hline First part of structural inspection & $\mathrm{K}$ & {$[2,3,3,3]$} & {$[2,2,3,3]$} & {$[2,2,2,2]$} & I & $0-0-2$ \\
\hline Second part of structural inspection & $\mathrm{L}$ & {$[1,1,3,4]$} & {$[1,1,3,4]$} & {$[1,1,3,4]$} & $\mathrm{H}-\mathrm{K}$ & $0-0-2$ \\
\hline Subcontracted painting & M & {$[1]$} & {$[1,1,2,2]$} & {$[1]$} & $\mathrm{L}$ & $0-0-0$ \\
\hline Second part of mechanical inspection & $\mathrm{N}$ & [1] & {$[1,1,1,2]$} & [1] & $\mathrm{F}$ & $1-1-0$ \\
\hline Assemble helicopter parts & $\mathrm{O}$ & [1] & {$[1]$} & [1] & G-J-M-N & $2-1-0$ \\
\hline Finishing before fly test & $\mathrm{P}$ & [1] & {$[1]$} & [1] & E-O & $1-1-0$ \\
\hline Test before delivering helicopter & Q & [1] & [1] & [1] & $\mathrm{P}$ & $1-0-0$ \\
\hline Possible additional work on helicopter & $\mathrm{R}$ & {$[1,2,2,3]$} & {$[1,2,2,3]$} & {$[1,2,2,3]$} & Q & $1-1-0$ \\
\hline
\end{tabular}

Table 1: Example of real projects data in MRO.
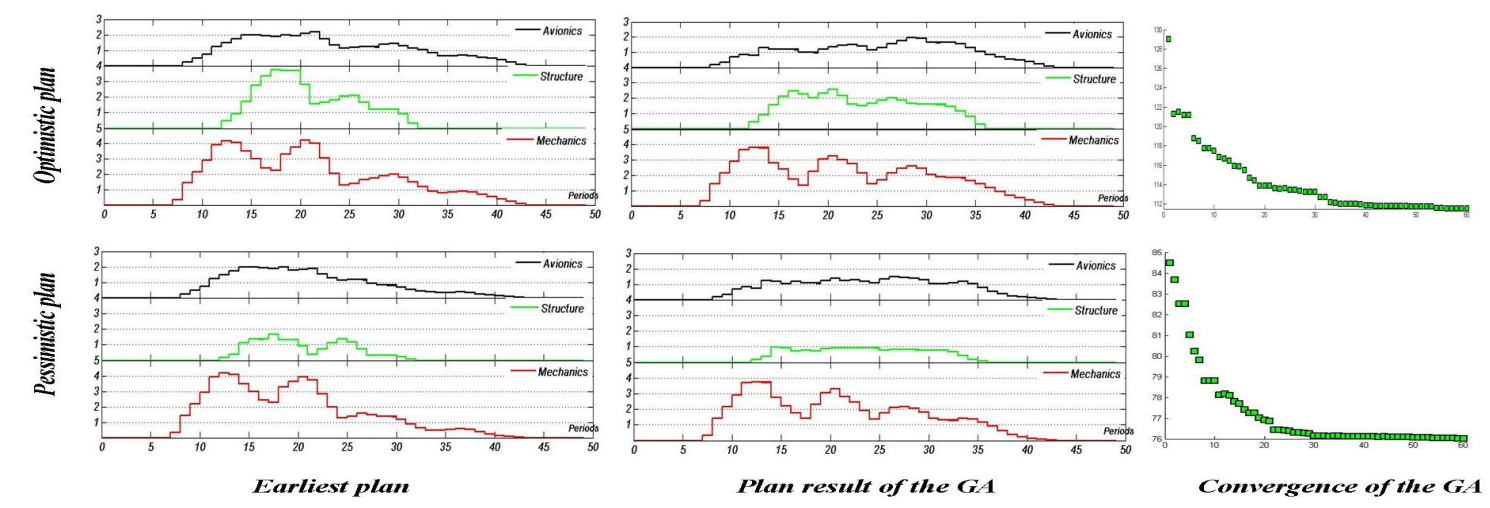

Figure 10: The earliest and the optimal (pessimistic and optimistic) workload plans

Addison-Wesley Longman Publishing Co., Inc., Boston, MA, USA, 1989.

[6] E. W. Hans and W. Herroelen and R. Leus and G. Wullink, A hierarchical approach to multiproject planning under uncertainty, Omega, $35: 563-577,2007$.

[7] M. Hapke and R. Slowinski, A DSS for resource constrained project scheduling under uncertainty. Journal of Decision Systems, 2:2:111117, 1993.

[8] M. Hapke and R. Slowinski, Fuzzy priority heuristics for project scheduling, Fuzzy Sets Systems, 83:3:291-299, 1996.

[9] M. Inuiguchi and M. Sakawa and Y. Kume, The usefulness of possibilistic programming in production planning problems, International Journal of Production Economics, 33:1-3:45-52, 1994.

[10] S.-J. Chen and C.-L. Hwang, Fuzzy multiple attribute decision making: Methods and applications, Springer, New York, 1992.

[11] K. W. Kim and Y. S. Yun and M. J. Yoon and M. Gen and G. Yamazaki, Hybrid genetic algorithm with adaptive abilities for resourceconstrained multiple project scheduling, Computers in Industry, 56:2:143-160, 2005.

[12] G. Alfred L. and N.Rakesh, Fuzzy set theory applications in production management research: a literature survey, Journal of Intelligent Manufacturing, Springer Netherlands, 9:1:39$56,1998$.

[13] S.-S. Leu and A.-T. Chen and C.-H. Yang, A
Fuzzy Optimal Model for Construction Resource Leveling Scheduling, Canadian Journal of Civil Engineering, 26:673-684, 1999.

[14] M. Masmoudi and A. Haït, A Tactical model under uncertainty for helicopter maintenance planning, proceedings of the $8^{\text {th }}$ International Conference of Modeling and Simulation(MOSIM2010), 3:1837-1845, Hammamet, May 2010.

[15] A. Soltani and R. Haji, A Project Scheduling Method Based on Fuzzy Theory, Journal of Industrial and Systems Engineering, 1:1:70-80, 2007.

[16] L. A. Zadeh, Fuzzy sets, Information and Control, 8:338-353, 1965.

[17] L. A. Zadeh, Fuzzy sets as basis for a theory of possibility, Fuzzy Sets and Systems, 1:3-28, 1978.

[18] S.-L. Zhao and Y. Liu and H.-M. Zhao and R.-L. Zhou ,GA-Based Resource Leveling Optimization for Construction Project, Proceedings of the $5^{\text {th }}$ International Conference on Machine Learning and Cybernetics(IEEE2006), 2363-2367, Dalian, August 2006.

[19] Professional site, Www.realization.com

[20] R. A. Hahn and A. M. Newman. Scheduling united states coast guard helicopter deployment and maintenance at clearwater air station, florida. Computers and Operations Research, 35:6:1829-1843, June 2008.

[21] A. Sgaslink. Planning german army helicopter maintenance and mission assignment. Mainte- 
nance Report, Naval Postgraduate School Monterey CA, March 1994.

[22] B. Grabot and L. Geneste and G. R. Castillo and S. Vérot, Integration of Uncertain and Imprecise Orders in the MRPII Method, International Journal of Intelligent Manufacturing, 16:215-234, 2005.

[23] D. Dubois and H. Prade, The mean value of a fuzzy number, Fuzzy Sets and Systems, 24:3:279-300, December 1987.

[24] P. Fortemps, Jobshop Scheduling with Imprecise Durations: A Fuzzy Approach, IEEE, Transactions on fuzzy systems, 5, 557-569, November 1997.

[25] L. Sánchez and I. Couso and J. Casillas, Genetic Learning of Fuzzy Rules based on Low Quality Data. Fuzzy Sets and Systems 160:17, 2524-2552, 2009. 